\title{
Design about Control System of Omnidirectional Mobile Platform Based on CAN Bus
}

\author{
Quan-li Wei ${ }^{1}$,Jiu-yan $\mathrm{Li}^{2}$,Yan Feng ${ }^{3}$ \\ \{wql@qust.edu.cn ${ }^{1}$,qd_ljy@163.com ${ }^{2}$,fywmh@163.com ${ }^{3}$ \} \\ School of Information Science and Technology, Qingdao University of Science and Technology, \\ Qingdao ${ }^{1,2,3}$
}

\begin{abstract}
In some traditional robot control systems, the communication between all kinds of sensors and CPU controllers is mostly based on the serial communication way which is difficult to adapt to the complex location environment in the industrial field. In order to solve this problem, through field experiments, it is found that CAN bus can be adaptable to severe environment due to its advantages of real-time communication and strong electromagnetic interference resistance. Based on this, this paper proposes a control system of omnidirectional mobile platform based on CAN bus, through the analysis of the motion of the angular velocity of the mobile platform, using CAN bus to collect the motor speed information and the fuzzy PID control algorithm to determine the precise motor speed, realizing high precision platform walking control. The results show that the system can carry out the work of material handling and assembly tools in a safe and normal way.
\end{abstract}

Key words: CAN bus, STM32, Omnidirectional mobile platform, Fuzzy PID control

\section{Introduction}

The omnidirectional mobile platform is a form of mobile robot. Its operating principle is to use specially designed Mecanum wheel to make the transport platform travel straightly, transversely and diagonally, etc. arbitrarily on two-dimensional plane. To improve system stability and reduce error rate of data, the paper proposes a new control system scheme for omnidirectional mobile platform, which adopts CAN bus technology and combines with fuzzy PID control algorithm, and adopts wireless remote control to control synchronously the motors of mobile platform, so as to apply it to high-precision mobile transport safely and accurately. 


\section{General design of system and critical technology}

\subsection{General design of system}

The CAN bus-based ${ }^{[1]}$ control system for omnidirectional mobile platform is composed of controller, 4 servomotors and motor driver connected to CAN bus. The modules are connected by CAN bus into a complete control system, and can transfer information in real time between each other. The general diagram of the system is as shown in Fig.1.

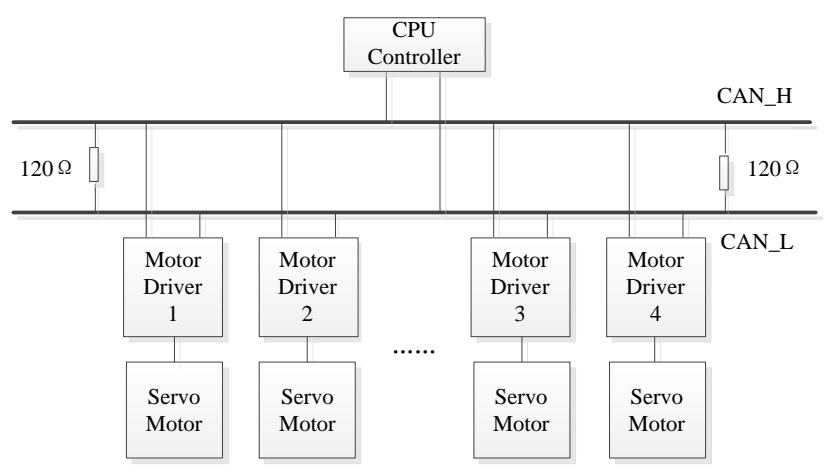

Fig. 1. The framework of control system

The operation process of the system is as follows: the system is electrified and reset, the CAN bus interface receives the control instruction message from platform controller, then broadcasts it to each motor driver, which filters the message identifier to obtain corresponding instruction to drive the corresponding motor to rotate. In the meanwhile, when the bus idles, and the motor driver transmits data to bus, the CAN transceiver transmits the data message to platform controller or other motor drivers.

\subsection{CAN bus}

The frame format ${ }^{[2]}$ adopted by message transmission for CAN bus differs according to the length of identifier field, and is divided into standard frame containing 11-bit identifiers and extended frame containing 29-bit identifier. As this communication system only needs to control 4 motors, standard frame containing 11-bit identifiers is adopted to accelerate the communication velocity.

In this system, each motor module node has an exclusive address code, according to which, the CAN bus transmits control instruction. In the control system for motor, its address code for master node is 30 , and the address codes for 4 controlled motor are 31, 32, 33, 34 respectively. 
The data frame in CAN control system mainly transmits data from transmitting terminal to the receiving terminals. Wherein, the data field contains 8 bytes. Wherein, the control instruction protocol is as shown in Table 1, and responses instruction protocol is as shown in Table 2 .

Table 1. Protocol of CAN bus control instruction

\begin{tabular}{ccc}
\hline Byte & Data & \multicolumn{1}{c}{ Explanation } \\
\hline Data1 & 0x00 & Motor velocity (low 8-bit) \\
Data2 & 0x00 & Motor velocity(high 8-bit) \\
Data3 & $0 x 00$ & Rotation direction of motor \\
Data4 Data8 & & Reserved \\
\hline
\end{tabular}

Table 2. Protocol of CAN bus response instruction

\begin{tabular}{ccc}
\hline Byte & Data & Explanation \\
\hline Data1 & 0x00 & Battery voltage \\
Data2 & $0 x 00$ & Battery's residual percentage \\
Data3 & $0 \times 00$ & Running velocity (low 8-bit) \\
Data4 & $0 x 00$ & Running velocity (high 8-bit) \\
Data5 & $0 \times 00$ & Motor velocity (low 8-bit) \\
Data6 & $0 \times 00$ & Motor velocity (high 8-bit) \\
Data7 & $0 x 00$ & State of rotation direction \\
Data8 & $0 \times 00$ & Fault code \\
\hline
\end{tabular}

\section{Design of fuzzy PID controller}

The paper establishes a kinematic theoretical model for omnidirectional mobile platform based on Mecanum wheel, thereby providing the theoretical references for control algorithm of omnidirectional mobile platform. Based on this, the fuzzy PID control algorithm is used to accurately control the rotary velocity of each wheel, guarantee the accuracy and stability of velocity and direction of mobile platform.

\subsection{Kinematic theoretical analysis on mobile platform}

The global coordinate $O_{x y}$ with platform center $O$ as origin as shown in Fig.2 is established. On the plane, the general angular velocity of platform is $\omega$, general movement velocity is $v$, and its components on $\mathrm{X}$-axis and $\mathrm{Y}$-axis are respectively $v_{x} 、 v_{y}$, represented 
by $\left[\begin{array}{lll}v_{x} & v_{y} & \omega\end{array}\right]^{T}$ here. Set the hub radius of Mecanum wheel as $\mathrm{R}$, and set the distance from wheel center to $\mathrm{X}$-axis and $\mathrm{Y}$-axis as $l_{1} 、 l_{2}$ respectively. The angular velocity by which the wheel rotates around axle is $\omega_{i}$, velocity of roller is $v_{g i}$, offset angle $\alpha$ between hub shaft and rotary shaft of roller is $45^{\circ}$.

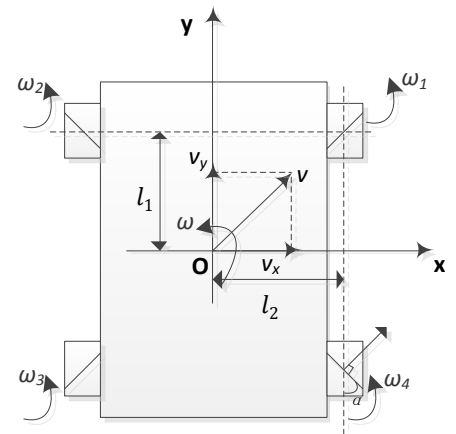

Fig .2. Schematic diagram of Mecanum wheel speed

With wheel 4 as an example, the velocity of wheel center in global coordinate system is:

$$
v_{4}=\left[\begin{array}{l}
v_{x} \\
v_{y}
\end{array}\right]=\left[\begin{array}{cc}
0 & -\sin \alpha \\
R & \cos \alpha
\end{array}\right]\left[\begin{array}{c}
\omega_{4} \\
v_{g 4}
\end{array}\right]
$$

As the wheel is integrated with omnidirectional mobile platform, the velocity of wheel 4 is obtained according to velocity of omnidirectional mobile platform:

$$
\begin{aligned}
& v_{4}=\left[\begin{array}{lll}
1 & 0 & l_{1} \\
0 & 1 & l_{2}
\end{array}\right]\left[\begin{array}{l}
v_{x} \\
v_{y} \\
\omega
\end{array}\right] \\
& {\left[\begin{array}{cc}
0 & -\sin \alpha \\
R & \cos \alpha
\end{array}\right]\left[\begin{array}{l}
\omega_{4} \\
v_{g 4}
\end{array}\right]=\left[\begin{array}{lll}
1 & 0 & l_{1} \\
0 & 1 & l_{2}
\end{array}\right]\left[\begin{array}{c}
v_{x} \\
v_{y} \\
\omega
\end{array}\right]}
\end{aligned}
$$

From equation 3, the mapping relation between Mecanum wheel 4 and platform velocity on plane $O_{x y}$ is obtained as follows:

$$
\omega_{4}=\frac{1}{R}\left[\begin{array}{lll}
\cot \alpha & 1 & l_{2}+l_{1} \cot \alpha
\end{array}\right]\left[\begin{array}{c}
v_{x} \\
v_{y} \\
\omega
\end{array}\right]
$$

Similarly, the inverse kinematics equation of omnidirectional mobile platform ${ }^{[3]}$ is: 


$$
\left[\begin{array}{l}
\omega_{1} \\
\omega_{2} \\
\omega_{3} \\
\omega_{4}
\end{array}\right]=\frac{1}{R}\left[\begin{array}{ccc}
-1 & 1 & l_{2}+l_{1} \\
1 & 1 & -l_{2}-l_{1} \\
-1 & 1 & -l_{2}-l_{1} \\
1 & 1 & l_{2}+l_{1}
\end{array}\right]\left[\begin{array}{l}
v_{x} \\
v_{y} \\
\omega
\end{array}\right]=J\left[\begin{array}{c}
v_{x} \\
v_{y} \\
\omega
\end{array}\right]
$$

Wherein, $\mathrm{J}$ is Jacobian matrix of kinetics equation of omnidirectional mobile platform, $\alpha$ is $45^{\circ}$.

As no element in this matrix is 0 , the rank of this Jacobian matrix $r(J)=3$, i.e. the matrix has no singular solution, so there is one-to-one correspondence between the rotation velocity of four wheels and mobile platform, indicating the system can realize omnidirectional movement.
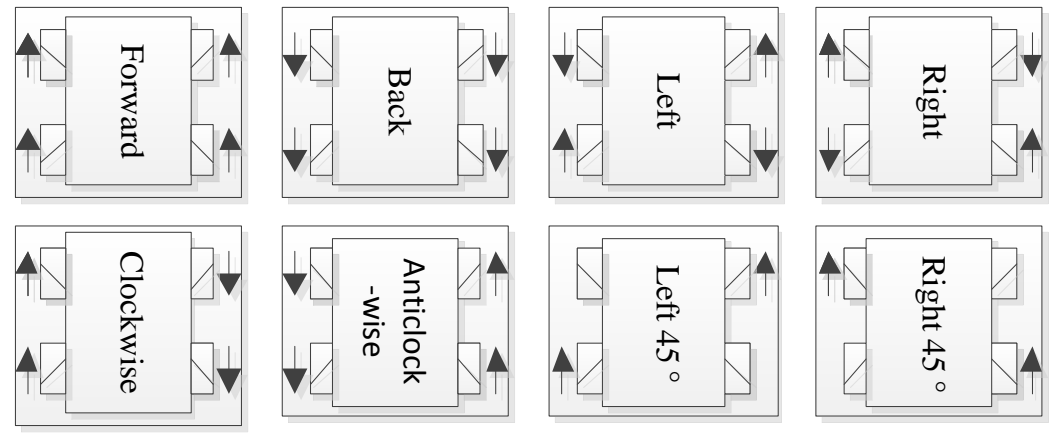

Fig. 3. Schematic diagram of omnidirectional mobile platform moving direction

According to different given rocking degrees of $\mathrm{x}, \mathrm{y}, \mathrm{z}$ in handle, $v_{x} 、 v_{y} 、 \omega$ with different values can be obtained, thereby controlling the motion of mobile platform in any direction. The general motion direction is as shown in Fig.3.

The movement of omnidirectional mobile platform is determined by the rotation velocity of motor and rotation direction. In the control process of omnidirectional mobile platform, the rocking degree is transmitted via wireless network to the mobile platform controller, which converts it into the angular velocity of each wheel. Then the corresponding parameters are transferred to motor driver via CAN bus network, and the motor driver controls rotation of motor, thereby making the whole mobile platform vehicle move along required direction.

\subsection{Design of fuzzy PID controller}

Based on physical conditions, the system adopts fuzzy PID controller to regulate the error of motor velocity for omnidirectional mobile platform, and applies the fuzzy PID control algorithm ${ }^{[4]}$ combining fuzzy control and PID control to the control over servomotor, to make the control of CPU controller over servomotor highly precise and more flexible. Wherein, the design of fuzzy controller mainly comprises three steps: fuzzifying, fuzzy reasoning, defuzzying. 


\subsubsection{Fuzzifying of input/output variables}

The system adds a fuzzy controller ${ }^{[5]}$ to the traditional PID controller. The deviation value $e(e=r-y)$ between given rotation velocity $r(t)$ of motor and actual feedback rotation velocity $y(t)$ and change rate of motor velocity deviation $e c(e c=d e / d t)$ are taken as input variable and adjusted value $\Delta K_{P} 、 \Delta \mathrm{K}_{\mathrm{I}} 、 \Delta \mathrm{K}_{\mathrm{D}}$ for PID parameter fed back by optical electricity encoder is taken as output variable, to establish two-dimensional fuzzy PID controller, as shown in Fig.4.

The fuzzyfying is actually to map the actual value of input/output variable to the corresponding fuzzy discourse domain. The actual discourse domain $\{-70,70\}$ of $e$ in the system and actual discourse domain $\{-10,10\}$ of $e c$ are all fuzzily discretized into $\{-6,-4,-2,0,2,4,6\}$, and corresponding fuzzy subset is \{negative big, negative medium, negative small,0, positive small, positive medium, positive big\}, which is expressed by $\{\mathrm{NB}, \mathrm{NM}, \mathrm{NS}, \mathrm{O}, \mathrm{PS}, \mathrm{PM}, \mathrm{PB}\}$. The discourse domain of output variable can be obtained via multiple experiments. Wherein, the discourse domain of $\Delta K_{P}$ is $\{-1,1\}$, that of $\Delta \mathrm{K}_{\mathrm{I}}$ is $\{-3,3\}$, and that of $\Delta \mathrm{K}_{\mathrm{D}}$ is $\{-1,1\}$.

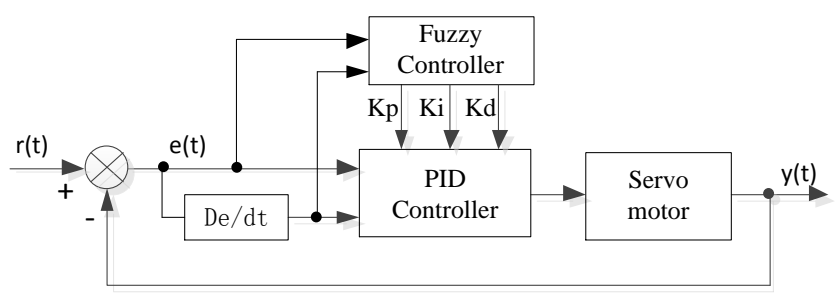

Fig. 4. The structure of fuzzy PID controller

The membership functions include triangular form, normal distribution type and Gaussian type, etc. Considering the requirement on timeliness of system design, the triangular membership function is adopted here to define the membership degree of fuzzy language variable. Wherein, the mathematic expression of triangular membership function is:

$$
\mu(x)= \begin{cases}\frac{x-a}{b-a}, & a<x<b \\ \frac{c-x}{c-b}, & b<x<c\end{cases}
$$

Where, the $\mathrm{b}$ value is set as $-6,-4,-2,0,2,4,6$. For triangular membership function, its overlapping coefficient ${ }^{[6]}$ is advisable to be about $0.3 \sim 0.6$, which can deliver a good effect. So, the setting is $(\mathrm{b}-\mathrm{a})=(\mathrm{c}-\mathrm{b})=1.1$. The function waveform of $e$ and $e c$ is as shown in Fig.5. 


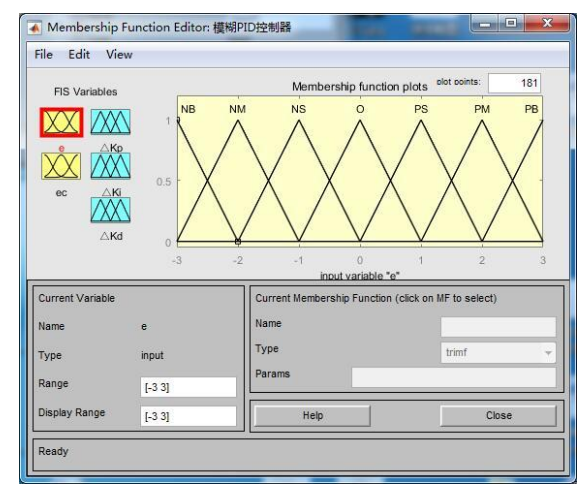

Fig.5. $e$ and $e c$ function waveform

\subsubsection{Fuzzy reasoning}

The technical knowledge of experts and accumulated practical experience show that the too small proportion factor $\Delta K_{P}$ slows down the corresponding velocity of system and lowers the dynamic performance; too small integration coefficient $\Delta \mathrm{K}_{\mathrm{I}}$ makes it difficult to eliminate static errors of system, which influences accuracy of adjustment of system; too small differential coefficient $\Delta \mathrm{K}_{\mathrm{D}}$ lower the stability of system. Based on this, the corresponding fuzzy rule can be established to effectively enhance robustness and stability of system and provide powerful guarantee for safe operation of omnidirectional mobile platform. The concrete form of If $\left(e\right.$ is NB) and ( $e c$ is NS),Then $\left(\Delta K_{P}\right.$ is PM)、 $\left(\Delta \mathrm{K}_{\mathrm{I}}\right.$ is NM)、 $\left(\Delta \mathrm{K}_{\mathrm{D}}\right.$ is PM) can be used to establish corresponding fuzzy relation to obtain table of three fuzzy rules ${ }^{[7]}$.

The output quantity obtained by reasoning via above reasoning table of rotation velocity fuzzy control is just a fuzzy set instead of definite data. In the actual application, this output quantity must be a precise value, which entails defuzzying the output quantity. According to the practical situation of the system, the weighted average method is adopted to defuzzy the output quantity. The equation is as follows:

$$
v_{0}=\frac{\sum_{i=1} v_{i} k_{i}}{\sum_{i=1}^{m} k_{i}}
$$

Where, $v_{0}$ represents exact value of output quantity, $v_{i}$ represents the membership value defined by triangular membership function, $k_{i}$ represents the fuzzy value obtained from fuzzy rule.

According to equation 7, the exact values of increments $\Delta K_{P} 、 \Delta \mathrm{K}_{\mathrm{I}} 、 \Delta \mathrm{K}_{\mathrm{D}}$ for three PID parameters are obtained, and are added to the $K_{P} 、 \mathrm{~K}_{\mathrm{I}} 、 \mathrm{~K}_{\mathrm{D}}$ reserved last time, to obtain the newest $K_{P} 、 \mathrm{~K}_{\mathrm{I}} 、 \mathrm{~K}_{\mathrm{D}}$, then according to increment PID equation ${ }^{[8]}$ : 


$$
\Delta \mu_{k}=K_{p} \Delta e_{k}+K_{I} e_{k}+K_{D} \Delta^{2} e_{k}
$$

Where, $e_{k}$ is the deviation value of input at time $k$

$$
\Delta e_{k}=e_{k}-e_{k-1} ; \Delta^{2} e_{k}=\Delta e_{k}-\Delta e_{k-1} \text { 。 }
$$

The increment of motor's rotation velocity is solved, and then the rotation velocity of motor is modified, to finish precise control over the system.

Above presents kinetic analysis on omnidirectional mobile platform for CAN bus-based control system for omnidirectional mobile platform and introduction of establishment of CAN bus network structure and design of fuzzy PID control system. Following is mainly the design of software of this control system.

\section{Software design for control system}

To improve the system software's readability and convenience of software debugging, this communication system was developed and debugged in integrated development environment of Keil $\mu$ Vision 4 .

The master program of this control system is executed according to sequential structure and cyclically detects the completion of corresponding functions by each status bit. As shown in Fig.6, firstly, the initialization of system, CAN, timer and interruption is finished;

Wherein, the configuration CAN initialization ${ }^{[9]}$ includes following steps:

1) Enable CAN clock, and set multiplexing function of related pin bit;

2) Set the operation mode and baud rate of CAN, etc.;

3) Set the operation mode information of filter and activate filter;

4) Enable receive interruption, set interrupt priority level.

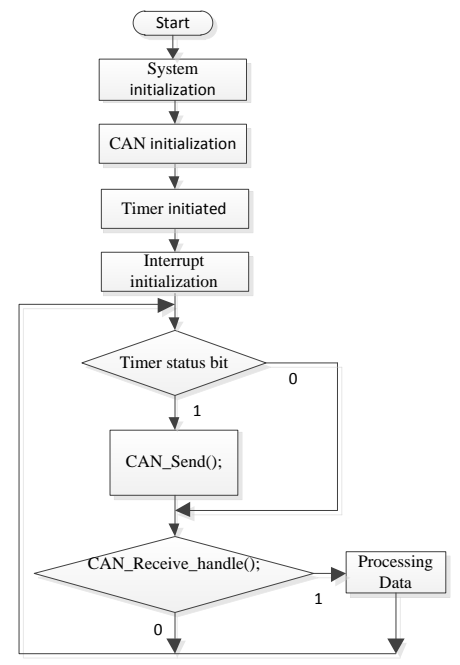

Fig. 6. Flow chart of CAN bus control system 
Then the major cycle of program is started to cyclically detect the status bit of system. Wherein, the transmit status bit is set in the interrupt service routine of timer. When the transmit status bit is triggered, frame information is transmitted. When a correct and complete piece of information is received, the receive status bit is triggered, and corresponding operation is conducted according to the frame content.

In this way, the control system adopts the extremely advantageous CAN bus technology and fuzzy PID control algorithm. The CAN bus technology does not only guarantee the velocity of data transfer, but also guarantees the reliability and stability of data transfer, while the fuzzy PID control algorithm guarantees the system stability and accuracy of control over platform, thereby making the omnidirectional mobile platform safely and stably operate in complicated field environment.

\section{$4 \quad$ Matlab simulation and analysis}

The feasibility of the fuzzy control system is verified by using the fuzzy logic toolbox in MATLAB. This PID controller's input variable is $e, e c$ and output variable is $\Delta K_{P} 、 \Delta \mathrm{K}_{\mathrm{I}} 、 \Delta \mathrm{K}_{\mathrm{D}}$. Those variables adopt the triangular membership function to define the membership degree of fuzzy language variable.

In the simulation analysis of the fuzzy PID controller, a sinusoidal perturbation wave with a period of $1 \mathrm{~s}$ and an amplitude of $2 \mathrm{~A}$ is added to the system. We get the response curve, as shown in Fig.7.

As can be seen in Fig.7, when the load switch on this system, in the process of system operation, the jumping is very small, and it can achieve the preset speed value in a very short time, and the reaction is very sensitive. Therefore, the system has strong robustness and can adjust the motor speed when the load is disturbed. Through simulation results, we can see that the fuzzy PID algorithm used in this system accords with the design requirements.

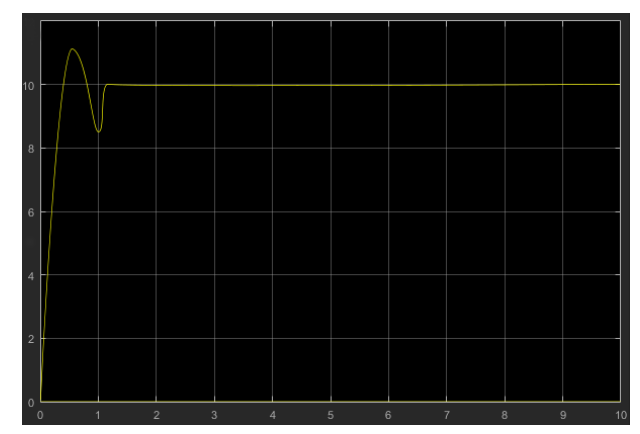

Fig.7. Response curve 


\section{Summary}

The field test showed that the CAN bus-based control system for omnidirectional mobile platform can guarantee the platform travels straightly, transversely and diagonally, etc. arbitrarily and finishes various rotations safely, flexibly and stably on two-dimensional plane under control of wireless remote controller. In the test process, the motor's rotation velocity of platform meets the given requirements on rotation velocity, and can regulate the platform velocity in different grades, thereby improving the stability of field operation of platform.

\section{References}

[1]Kuanming Wu. Design of CAN Bus Principle and application system[M]. Beijing:Beihang University press(2002).

[2]Yuntao Rao, Jijun Zou, Jinhong Wang. The principle and application of CAN field bus technology[M].Beijing:Beihang University press.24-30(2007).

[3]Boweng Chen,Chuankang Fan,Ji He.Key Technology Research about omnidirectional Mobile Platform Based on Mecanum Wheel[J],Dongfang Electric Review.27(04):7-11(2013).

[4]Jian Li.Research on Motion Control of Remote Operated Vehicle Based on Fuzzy PID Control[D].Dalian University of Technology(2016).

[5]Xiaoju Yang,Wei Zhang,Hongwei Gao. Study on obstacle avoidance of mobile robot based on fuzzy control[J]. Transducer and Microsystem Technologies. 36(03):51-54(2017).

[6]Jing Li,Wenbin Li.Study on the path planning of mobile robot based on fuzzy control [J]. Journal of Chinese Agricultural Mechanization. 36(1):272 274+278(2015).

[7]Zhongmin Zhang, Renhui Zheng. Control algorithm of mecanum-wheel mobile platform based on fuzzy PID[J]. Applied Science and Technology.44(06):53-59(2017).

[8]Yuexian Zhang.Simulation of fuzzy PID control system for brushless DC motor speed[J].Electrotechnical Application. 35(24):24-29(2016).

[9]Weiran Hua.Design and Implementation of Stepper-motor Control System Based on CAN Bus[J]. Mechanical engineering and automation, 2017(03):166-168(2017). 